\title{
Interdisciplinary Collaboration in Chinese Higher Education: Approaches, Problems and Possible Solutions
}

\author{
Caihong Zhang \\ College of Humanities and Foreign Languages, Xi'an University of Science and Technology, 58 \\ SouthYantaRoad, Xi'an, Shanxi Province, China \\ zhangcaihonghappy@163.com
}

Keywords: Interdisciplinary collaboration; Chinese higher education; Research universities

\begin{abstract}
In the context of encouraging interdisciplinary collaboration in higher education, many Chinese top universities have made efforts in reducing the barriers to conduct research and teaching that involve members from multiple disciplines. This paper examines some interdisciplinary approaches applied in Chinese top universities, aiming at giving suggestions to solve the problems appeared in the interdisciplinary cooperation process in Chinese higher education. Issues covered in the paper include but not limited to interdisciplinary research, faculty development, curriculum designing and student cultivating.
\end{abstract}

\section{Introduction}

With boundaries among disciplines becoming more and more blurring, producing integrative knowledge has been paid more attention in education. Numerous facts show that interdisciplinary collaboration is a more effective way for scientific research, faculty development and students training. That's why developed countries are attaching increasing importance to interdisciplinary activities in higher education institutions, especially in the research universities. Large organizations like National Science Foundation and the Association of American Universities have given priority on multidisciplinary activities, too. Accordingly, a campaign has been launched in the US on interdisciplinary concentration by making efforts in large-scale research programs, innovative curricula design, and cooperative teaching etc. The purpose of doing so is to set up a collaborative force in the cutting-edge areas for the country so as to improve economy development.

Following up these practices concerning interdisciplinary innovation, many studies have been conducted to evaluate the worthiness as well as flaws shown up in the crossing disciplinary movement with the purpose of improving it and helping scholars to meet the challenges faced in implementing process. On balance, most of these studies confirmed people's confidence in these collaborative activities by showing the great results researchers, educators and students have achieved. Meanwhile, suggestions have been given too on how to solve the problems rising in practice.

\section{Transformation Brought about by Interdisciplinary Campaigns}

Scholars of higher education are in the consent that interdisciplinary activities have not only changed people's perspectives on research and knowledge, but also transformed administration concept and cultures at higher educational institutions. According to Karri A. Holley, initiatives of interdisciplinary activities involve five key elements: senior administrative support, collaborative leadership, flexible vision, faculty and staff development and visible actions [1]. Surveys conducted by other scholars like Lisa Benton-Short, Kathleen A. Merriganand Creso M. Sa. at research universities in the United States came to the closing conclusion [2,3]. These universities and colleges have some in common despite their individual characteristics in the interdisciplinary experiments. First, a platform is usually established where the interdisciplinary programs are conducted which may be a center, a department, a committee or a union. Second, senior administrative support, financially or administratively, is a must in the crossing disciplinary 
organizations. Third, interdisciplinary organizations have their own leaders whose responsibility is to organize research programs and coordinate other affairs. For instance, a new office of full-time vice provost is usually created for interdisciplinary endeavors in Duke University in the United States [1]. Certainly, interdisciplinary programs in various forms like research projects, academic programs, curriculum redesign, group teaching, public engagement ect. are carried out beyond the boundaries of traditional departments or schools. Then, reforms of evaluating and rating systems about achievements and fund allocation among faculty are conducted as a result of crossing disciplinary innovation. Since the boundaries of department have been torn down, faculty appointment, promotion and administration as well as campus culture based on department have been transformed, too.

\section{Interdisciplinary Collaborations in Chinese Universities}

Theoretical Studies. Inspired by the remarkable achievements made in western countries in higher education, more and more Chinese scholars started to pay attention to researching, teaching and administrative practices in top foreign universities and introduce those western ideas and modes to China, hoping to improve Chinese higher education. Articles published in academic journals in this field break down into two major kinds: one is introducing on a single aspect of the interdisciplinary collaboration by taking top foreign universities and colleges as examples. For instance, studies conducted on curriculum design or students cultivation or project undertaking or organization building from the interdisciplinary perspective are seen in this kind $[4,5,6]$. The other kind is examining Chinese higher education status quo in contrast to those of the developed countries so as to explore some applicable interdisciplinary modes in Chinese higher education institutions [7,8].

Considering the theoretical research on crossing disciplinary cooperation done so far in higher education in China, we can arrive at the conclusion that all-sided surveys to a greater degree are still lacking. Or in other words, Chinese scholars have not much systematic examinations in interdisciplinary collaboration. However, in America, such explorations have been done for decades and lots of experiences and evaluations also have been achieved to perfect it $[1,3,9]$.

Exploration and Experiment in Practice. Like in America, Britain and other countries, Chinese government agencies like Ministry of Education (MOE) declared support for cooperation crossing disciplines in higher education by supplying financial help. In 2012, the document issued by MOE titled "Suggestions on Improving the Overall Quality of Higher Education" stressed the importance of building key labs, engineering technology and research centers. Under the call of this, some top research universities in China promoted the movement of interdisciplinary collaboration.

According the investigation of Chinese scholars Guo Zhonghua, Huang Zhao and Zou Xiaodong from Zhejiang University (2008), Feng Lin and Zhang Zhixiang from Da Lian University of Technology (2014), universities in China conduct interdisciplinary research via four major approaches:

Building large laboratories or research centers at universities which are in the charge of certain schools or universities despite the names of "state" or "national" rank. These labs are usually labeled as the "the Key Lab" or "the National Key Lab", owned and funded directly by government agencies. State-level projects involved in two or more disciplines cooperation are being organized and conducted here, most of which are funded by the Natural Science Foundation Committee (NSFC), the Ministry of Science and Technology (MOST) or MOE. For instance, Tsinghua National Laboratory for Information Science and Technology, State Key Joint Laboratory of Environmental Simulation and Pollution Control at Tsinghua University and Beijing International Center for Mathematical Research, State Key Laboratory for Structural Chemistry of Unstable and Stable Species at Peking University are such kinds. By the end of 2016, hundreds of labs and centers have been built in Chin aiming to assist research crossing disciplines. Tsinghua University alone has 137 research institute authorized by the government by June 30, 2016.

Building of independent research organizations as centers or unions at universities to provide help for cooperation crossing disciplines. Supported financially by universities, these organizations 
usually belong to an interdisciplinary school. For example, Center for Quantum Information (CQI) and Institute for Theoretical Computer Science (ITCS) from Tsinghua Universities are typical examples of these organizations. The Academy for Advanced Interdisciplinary Studies from Peking University also contains similar independent interdisciplinary organizations like Centre for Environment and Health, Center for Quantitative Biology etc. Accordingly, leaders of the centers or institutes are in charge of collaborative issues which may include the faculty recruit, fund applying, research coordinating during cooperation etc. Meanwhile, these kinds of organizations are, on most occasions, the base for university teaching, researching and production. By June 30, 2016, Tsinghua University has eight interdisciplinary organizations and Peking University has seven.

Research Cooperation with local government, enterprises and overseas organizations to realize interdisciplinary collaboration. Organizations jointly built by schools from universities and local government are aiming at becoming technology transfer bases to improve local economic development. For instance, Tsinghua University-Hefei Public Safety Research Institute in 2013 and Tsinghua-Suzhou Automotive Research Institute in 2011 are this kind. Another organization called the University-Industry Cooperation Committee (UICC) of Tsinghua University built in 1995 with the purpose of integrating industry, education and research to promote the industrialization of high-tech achievements and provide multi-dimensional, diversified services to assist enterprises in solving problems in technology and management are the platform available for cooperation from different disciplines. China Telecom, China Huaneng Group Corporation, and Huawei, Sichuan are only a part of the enterprises cooperation with Tsinghua University. Moreover, some overseas joint organizations have also been seen establishing between Chinese research universities and those of other countries, such as the Tsinghua-UC Berkeley Shenzhen Institute (TBSI) in 2015, are good examples for interdisciplinary research collaboration between China and the U.S. in the area of top-level higher education.

"Discipline groups" in Project 211 and Project 985. For the Project 211, disciplines which may interweave in research were grouped together and funded by Chinese MOE directly. However, many disciplines in these groups haven't much research collaborations in practice and the funds usually were used by different discipline studies individually. However, the establishing of new interdisciplinary platforms for science and technology innovation in Project 985' second stage is being undergone now in China.

\section{Problems and Possible Solutions to Chinese Interdisciplinary Transformation}

Implementation of interdisciplinary collaboration in higher education institution has caused many positive changes, some of which are in the interdisciplinary teaching and research, some are from the level of school administration, leadership, faculty and education actions. Campus culture and concept of education have been transformed too. However, problems appeared too in Chinese universities during these interdisciplinary experiments because of the comparatively short exploration history.

First, the administrative changes happened in Chinese universities are more about leaders' responsibilities for implementation of research projects, priorities shaping, fund supporting than faculty organizing and coordinating. Accordingly, small groups without enough members became an obstacle for some interdisciplinary research. And for the large groups, tensions among members in and out of work are not paid enough attention.

Second, when it comes to student education, the independent interdisciplinary institute needs to do more to make the interdisciplinary talents cultivation work efficiently. Lots of universities do not have a mature systematic blueprint about undergraduate training. Most of the courses are designed for students from one single major or field with teachers from one school or department. Core curriculum is lacking and cannot meet the demand of students in many interdisciplinary institutes. Collaborations involved in nature and social science are very rare in curriculum.

Third, despite the fact that many universities in China have built centers and institutes to enhance cooperation with members from local or enterprises so as to provide service and members training, the training and further development for school faculty itself is often lacking. Many faculty 
members in interdisciplinary institutes are from different departments/schools and need to learn skills of association and negotiation which is important in collaborative work. Besides, interdisciplinary work demands faculty constant learning to achieve expertise updating. Yet, professional development is usually underestimated both within and out of disciplinary boundaries.

Evaluation of faculty members' academic achievement is another problem. Most interdisciplinary projects are jointly done by researchers from different schools; therefore it's hard for faculty members to get individual credit because of the unclear allocation. And it's difficult for them to get professional recognition as well when the rating system in China is still based on disciplinary peer evaluation within a discipline. As a result, professional promotion seems be especially hard for the interdisciplinary scholars.

Interdisciplinary cooperation in China has a much shorter history compared with those in the western countries. Even the top universities like Tsinghua University and Peking University only have about three decades of experience in it. Problems here are more complicated and need different solutions considering the different educational situations. For the university administrative group, they need to give more support and rights to the leaders of interdisciplinary schools, and more faculty members need to be encouraged to work crossing boundaries. For the interdisciplinary organizations, curriculum is a core for students training, teachers' collaboration and research conducting, thus more efforts need to be made to make it work. Then, new faculty evaluation models should be created urgently to improve the old policies, for which many experiences from abroad may be consulted. Besides, the number of Chinese university which has interdisciplinary schools or departments is far too small. More collaboration crossing disciplinary boundaries need to be encouraged.

\section{Conclusion}

Interdisciplinary collaboration in Chinese research universities discussed above represent some situations only. Different schools may have their individual modes, facing different challenges and having different problems, thus variety in application of strategies is quite for sure. No matter how different the situation might be, for each university, administration support, curriculum creation, faculty's development and professional achievement rating is the issues that always matter as long as the interdisciplinary collaboration is concerned. To Chinese higher education, how to balance cooperation crossing disciplines at institutional level and protect faculty's academic credit while the rating system still does not favor crosscutting collaboration, how to gain more support from the senior administration to the lower levels of interdisciplinary organizations while making sure the mechanism work efficiently, and how to encourage more scholars to interdisciplinary research and be able to provide them with constant professional development chance are the problems need to tackle with more urgency.

\section{Acknowledgements}

This article is funded by Shaanxi Soft Science Research Program (2015KRM129).

\section{References}

[1] A. Karri Holley. Interdisciplinary strategies as transformative change in higher education. Innov High Education 34(2009) 331-344.

[2] Lisa Benton-Short, A. Kathleen Merrigan. Beyond interdisciplinary: how sustainability create support unities for pan-university efforts. J Environ Stud Sci. (2015).

[3] M. Sa. Creso Interdisciplinary strategies' in U.S. research universities. High Education 55 (2008) 537-552. 
[4] Di Wu. On the integrative science at top universities: interdisciplinary curriculum practice at Princeton University. Heilongjiang Researches on Higher Education. 10 (2012) 65-68.

[5] Yong Chen etc. Research on organizational model of improvement in interdisciplinary research: based on analysis about the Biox-program in Stanford University and implications. Studies in Science of Science. 3(2010) 346-350.

[6] Jayu Deng. Interdisciplinary course design in American universities. Education Theory Study. $23(2015) 15$.

[7] Feng Lin, Zhixiang, Zhang. Analysis of the basic mode and operating mechanism of interdisciplinary research in Chinese universities. Heilongjiang Education (Higher Education Research \& Appraisal 222 (2012) 55-57.

[8] Wen Shaobao. Pre-warning function of interdisciplinary social science research in HEIs: a case study of ISR in University of Michigan. Modern University Education 2 (2012) 63-68.

[9] J. Shirley Vincent. Timmons Roberts, and Stephen Mulkey. Interdisciplinary environmental and sustainability education: islands of progress in a sea of dysfunction. J Environ Stud Sci. 04, July, (2015). Published on line.

[10]Zhonghua Guo, Huang Zhao, and Xiaodong Zou. Problem of the Realization of interdisciplinary Orgnizations and its Countermeasures. Science \& Technology Progress and Policy 25 (2008) 183-186. 